\title{
Delayed auditory feedback with delayed sounds varying in duration
}

\author{
PETER HOWELL \\ University College London, London, England \\ and \\ DAVID J. POWELL \\ Moorfields Eye Hospital, London, England
}

\begin{abstract}
Speakers frequently report using strategies to circumvent the disruptive effects of delayed speech feedback. Often the strategies adopted are inconsistent with the traditional account, which maintains that the disruption occurs because speakers try to use the delayed speech for feedback control, even though it is now delayed and therefore is inappropriate for this control purpose. One strategy that subjects report using is to wait for the delayed sound to stop before proceeding with more speech. A prediction that follows is that disruption at a particular delay will depend upon the length of the delayed sound. We tested and confirmed this prediction by truncating the delayed speech and assessing the disruption this caused. The same effect occurred when a nonspeech sound was substituted for the delayed speech. The implications of adopting this strategy for coping with the effects of delayed auditory feedback, and the equivalent results obtained with speech and nonspeech, are discussed with respect to alternative accounts about why such disruption might occur.
\end{abstract}

Speech control suffers if auditory feedback of the voice is delayed. The effects of delayed feedback include a reduction in speech rate and an increase in all types of speech errors. The time to read a set passage increases up to a delay of about $200 \mathrm{msec}$ and then decreases somewhat. Thus, the disturbance function across delays is in the shape of an inverted $U$. This $U$-shaped disturbance function also occurs with speech errors: error rate increases to a maximum at a delay of $200 \mathrm{msec}$ and then drops off. The pitch and level of the voice are affected, too, but behave somewhat differently: these measures increase up to a delay of $200 \mathrm{msec}$, but then the function levels off rather than decreases.

Such disturbances would occur if speakers normally listen to their speech to check that it is being produced correctly and continue to control speech in this manner even when the speech is delayed experimentally. In the latter case, the speech would not provide appropriate feedback to the speaker about his/her present articulatory actions, resulting in the observed disruption of speech control.

Black (1951) adopted this account to explain the disturbance noted with reading time and error rate: if a sequence of instructions corresponding to syllables is sent to the peripheral articulators, maximum disruption will occur when the delay is equal to the length of the syllable. At this delay, the speaker would be issuing instruc-

The first author was supported by the Medical Research Council of the United Kingdom. His address is: Department of Psychology, University College London, Gower Street, London, WC1E 6BT, England. tions for one syllable but would hear what was produced on the preceding one. Syllables are about $200 \mathrm{msec}$ long; thus, Black's account explains why maximum disruption occurs at a 200-msec delay, and supports the view that syllables are the basic segment issued during speech control.

There are a number of problems with this and similar interpretations of the effects of delayed auditory feedback (DAF). First, there is evidence that subjects use strategies that are inconsistent with an auditory-feedback account. Susceptibility to the effects of DAF is one example. Generally speaking, fluent speakers show less disruption than do less fluent speakers. Thus, males (Bachrach, 1964), young children (Siegel, Fehst, Garber, \& Pick, 1980), and speakers employing a language acquired late in life (MacKay, 1970) are more affected by DAF, and less fluent, than are female speakers, older children, and speakers employing their native language. The obvious conclusion to be drawn from these data, if it is assumed that DAF provides evidence for an auditory-feedback monitoring account of speech control, is that less fluent speakers rely more on auditory feedback than do their fluent counterparts, and thus are more disrupted when auditory feedback is interfered with experimentally.

However, Howell and Archer (1984) reassessed this conclusion. They reported that an individual's susceptibility to the effects of DAF could be affected by voluntary alterations to voice level; when voice level was increased, more disturbance occurred. Fluency is unlikely to vary markedly with voice level, and therefore is unlikely to underlie differential susceptibility to DAF. More- 
over, in Bachrach's (1964) study and when the children spoke at a normal rate in Siegel et al.'s (1980) study, fluency was confounded with voice level. In both of these studies, the least fluent speakers, who were found to be most susceptible to DAF, spoke louder, again suggesting that voice level is the factor that causes different degrees of susceptibility.

The data reported by Howell and Archer (1984) also suggest that these variations in the disruption caused by DAF are strategic, rather than inherently tied up with how different speakers use feedback. As an example, subjects often report that when they hear DAF, they raise their voice levels to increase the amount of feedback that is not delayed; this feedback arrives by bone conduction, leakage from the headphones, and so forth. This strategy is, of course, counterproductive, because the delayed sound, as well as the feedback received directly, is louder. However, the fact that the speakers alter their voice levels in an attempt to "beat" the experimental intervention indicates that these findings have no implications about how people use feedback in a normal speaking situation.

Another strategy that speakers report using in order to get around the disturbing effects of DAF is to produce the desired sound and then wait for the delayed sound to finish before they produce the next sound. This strategy would account for the observed time disturbance: The amount of time the speaker waits (and therefore the amount of additional time required to read a set piece) would be proportional to the extent to which the delayed sound "sticks out" beyond the produced segments. If "sticking out" occurs beyond the end of the syllable, then the amount of time to read the list would increase up to a delay equal to the length of a syllable.

If subjects use this strategy, it follows that for sounds with the same delay at onset, the increase in time required should be determined by the length of the delayed sound. If the sound starts at the same point in the time but is shortened, the speaker would have to wait a shorter length of time before starting the next syllable. Thus, if listeners employ this strategy, the amount of disruption should vary directly with the length of the delayed sound. If speakers employ this strategy, the form of the disturbance function would be a product of how the listener tries to circumvent the disruptive effects, not of how the speaker attempts to use the sound to control the voice.

Strategies that speakers employ are, then, one line of evidence that undermines an auditory-feedback monitoring account of speech control. A second line of evidence that argues against DAF's offering support for auditory feedback control is that the delayed signal need not be speech in order to produce the sort of disruption noted (Howell \& Archer, 1984; Howell, Powell, \& Khan, 1983). Thus, Howell and Archer (1984) reported that when a nonspeech sound was substituted for the delayed speech sound in a DAF task, time to read a set list of vowels (performance time, or PT) peaked at a delay of about $200 \mathrm{msec}$, as is usually found with speech. No difference was found in the PT function across delays between speech sounds and nonspeech sounds when the level at the headphones was the same. The nonspeech sounds could not be used for speech control, and so the disturbance must have occurred for some other reason.

Howell et al. (1983) proposed that the fact that disruption caused by speech and nonspeech is equivalent and peaks at a delay of about $200 \mathrm{msec}$ is due to the relationship between intensity changes at a segmental level and syllables (Fant, 1959). Under DAF, the modulation in intensity of the delayed signal is out of synchrony with the current speech. The speaker is producing one rhythm and hearing a different one, and this may cause disruption in performance. If this asynchrony between the rhythm produced and the rhythm heard is the cause of poor speech control, then, providing that the disrupting sound has the same rhythm as the delayed speech (as it does in the experiments of Howell \& Archer, 1984, and Howell et al., 1983), no difference would be expected between performance with speech and that with nonspeech.

According to Howell et al. (1983), maximum disruption occurs at a delay of $200 \mathrm{msec}$ because syllables are about $200 \mathrm{msec}$ in duration. Thus, at this delay, the delayed sound will begin at the point at which the current syllable is finishing. Howell et al. cited evidence to support the view that two rhythms that stand in this relationship are particularly difficult to perform. For example, hand-bell ringers have difficulty when they have to produce rings sequenced so that at the offset of a ring by one player, the ring of another player occurs.

Thus, the nonspeech substitution studies show that performance under DAF of speech is determined by the speech's producing a disruptive rhythm, and it is where the disruptive rhythm starts, not whether it is possible to use this sound for feedback control, that determines the disturbance of performance. The strategies employed to circumvent the effects of DAF (speaking louder or waiting for the bit that sticks out beyond the end of a syllable to finish) could be adopted by speakers whether the disturbing sound is speech or nonspeech.

In sum, subjects in DAF experiments employ strategies-such as choosing what voice level to use and waiting for the delayed sound to finish before starting the next- that are incompatible with feedback-monitoring explanations. Also, it appears that it is where the delayed signal occurs, relative to the end of each syllable, that determines the disruption of performance.

In the experiment reported here, the strategy of waiting for a sound to finish before starting the next was examined when the delayed sound was speech and when it was nonspeech; the time to read a standard list under different delays was measured for both speech and nonspeech when the duration of the delayed sound was adjusted so that it lasted for different lengths of time.

The following predictions were made concerning the disturbance function that relates delay to the amount of time to read a standard list: If speakers adopt the strategy 
of waiting until the delayed speech has ceased after each syllable spoken, then disturbance should vary with the length of the sound protruding beyond the end of each syllable. For a particular stimulus duration, the disturbance function should show maximal disruption when the delayed sound starts to increase in intensity at the point at which the current sound is dropping to its minimum. Thus, for all stimulus durations, a peak should occur at a delay of about $200 \mathrm{msec}$, because, as discussed earlier, the intensity of sounds is related to the structure of syllables. For a given stimulus duration, the height of the peak at a delay of around $200 \mathrm{msec}$ should decrease as duration of the delayed signal decreases. Finally, and again as detailed above, equivalent disturbance should be observed whether the delayed sound is speech or nonspeech. A traditional feedback account would predict that when the delayed sound is altered (i.e., when it is shortened, or when nonspeech is substituted for speech), it cannot be used for feedback control, so that the usual findings observed with DAF of speech-in particular, the disturbance function with a peak at a delay of about $200 \mathrm{msec}-$ should not occur.

\section{METHOD}

\section{Subjects}

The 16 subjects ranged in age from 18 to 25 . For 8 subjects, speech sounds were presented after a delay; the other 8 subjects heard nonspeech after the specified delay. In each group, 6 subjects were male and 2 female.

\section{Materials}

Lists of the vowels /i/ and / $\mathrm{u} /$ were employed. Each list consisted of a total of 50 vowels; $/ \mathrm{i} /$ and $/ \mathrm{u} /$ occurred 25 times each. The sequences were also controlled so that all possible 3-tuples occurred equally frequently over a list of 50 vowels. This ensured that no aspects of the lists made some easier than others. A total of 30 lists were prepared.

\section{Apparatus}

The experimental manipulations were the delay of a sound, the truncation of the delayed sound, and the substitution of a nonspeech sound for the normal-length or truncated speech sound.

The speech to be delayed was transduced by a Beyerdynamic microphone held $20 \mathrm{~cm}$ from the speaker's mouth. This speech was amplified, low-pass filtered at $3.5 \mathrm{kHz}$ ( $48 \mathrm{~dB}$ per octave), and led to a PDP $11 / 10$ computer, which was programmed to operate as a variable delay line. The computer delayed the speech by digitiz-

\section{STEPS IN THE CONSTRUCTION OF THE GATING WAVEFORM.}

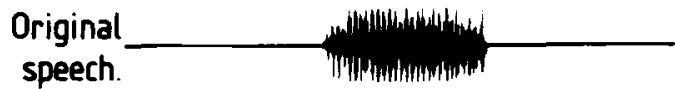

Original
speech
delayed.

Envelope(a).

Inverted

envelope.

Inverted and

delayed envelope (b)
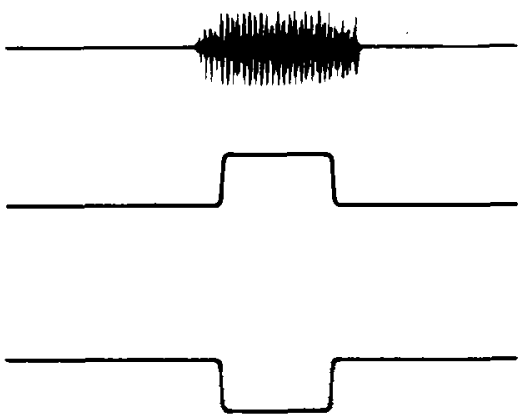

Gating function

$((a) \times(b))$.

Figure 1. Fow diagram indicating the steps involved in construction of the gating waveform employed in the experiment. 
ing it at a $10-\mathrm{kHz}$ rate and outputting the samples through one of the A/D outputs (also at $10 \mathrm{kHz}$ ) after the specified delay. The output was filtered (again at $3.5 \mathrm{kHz}$ and with a filter roll-off of $48 \mathrm{~dB}$ per octave), amplified, and led to the speaker's ears, where it was heard over AKG K120R headphones. The delays employed were $0,100,150,200,250$, and $300 \mathrm{msec}$.

The speech was truncated by creating a gating waveform from the delayed speech. The first step in creating the gating waveform was to obtain the syllable envelope. As already observed, intensity fluctuations in speech are associated with syllables: intensity starts to rise at the onset of the syllable, reaches a maximum at the syllable nucleus, and falls off at the end of the syllable. The envelopes of the syllables were obtained by passing the speech through a Fonema speech signal rectifier and low-pass filtering at $50 \mathrm{~Hz}$ (a roll-off of $48 \mathrm{~dB}$ per octave was used). For the envelope to be used as a gating function, the gate has to open at the point where the experimenter wishes to switch on the sound. In the present experiment, because the opening of the gate occurred at the delay speci- fied by the delayed speech output from the computer, the delayed speech was led to the envelope-detecting routine. The delayed speech was available from a parallel output in case a delayed portion of the speech was to be gated on and off (see below).

To produce the offset gating function, it is necessary to control when the offset occurs. Therefore, the envelope was obtained, as for the onset-gating function, and was then inverted with a purposebuilt unity-gain inverting amplifier. Thus, when the original gate opens (as specified by the intensity envelope of the syllable) the inverted gate closes, and vice versa. Now, if the inverted envelope is delayed relative to the intensity envelope to be used as the ongate, and the two gates are multiplied together, a gating function is obtained that is open for the duration specified by the time interval between the two envelopes. The offset gating function has to be delayed by the delay required before speech starts plus the duration that is required for the sound's onset. This was done by the computer, using the same logic as for a simple delay; the speech with the delay required for the offset gate was put out of a different

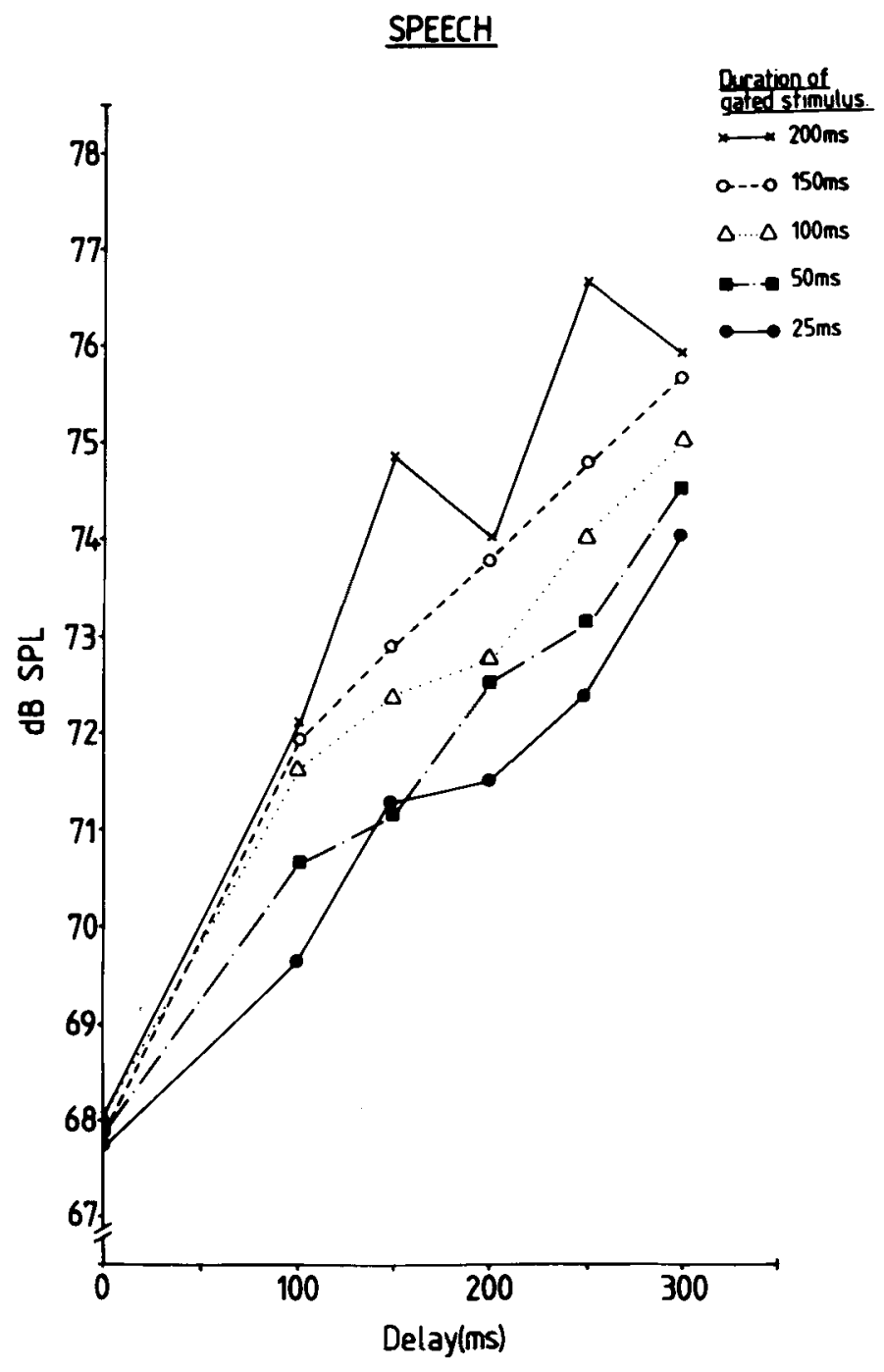

Figure 2. Mean SPL over subjects as a function of delay (in milliseconds) for the subjects who heard speech in the experiment. Delay is along the abscissa and SPL along the ordinate. Connected points come from conditions in which the delayed speech sound had the same duration (the duration can be identified from the symbol labeled in the inset). 
A/D converter. The delays on this channel were chosen so that sounds $200,150,100,50$, and $25 \mathrm{msec}$ in length occurred at all the delays. The steps employed in producing the gating waveform are summarized in Figure 1, along with diagrams indicating what each step achieves in producing the gating waveform.

Once the gating function is available, the speech can be switched on for the required length of time by multiplying the gate with the speech. The speech and gate at onset always had the same delay in the present experiment. Thus, if speech was to be delayed by $200 \mathrm{msec}$ and to last for $100 \mathrm{msec}$, the onset envelope was delayed by $200 \mathrm{msec}$ and the offset envelope was delayed by $300 \mathrm{msec}$ to produce the gate. The speech was then delayed by $200 \mathrm{msec}$, switched on after the 200 -msec delay, and switched off $100 \mathrm{msec}$ later by the gating envelope.

To substitute a nonspeech sound for the delayed speech and to give it a specified duration, the gate was created in exactly the same way as for the speech. The difference was that this gating envelope was used to switch on a nonspeech sound. The nonspeech sound was a triangular wave from a signal generator and the generator was running at a constant $100 \mathrm{~Hz}$ throughout. The speech and nonspeech sounds were calibrated by sustaining a vowel at $70 \mathrm{~dB}$ SPL into the equipment and adjusting the gain.

\section{Procedure}

The subjects were assigned at random to either the group that heard speech or the group that heard nonspeech. Apart from the nature of the sounds, the procedure for the two groups was exactly parallel. Order of delays was randomized across subjects. Once the order of delays was established, the subject would perform in all conditions with that delay, but the sounds involved would vary in duration. The duration orders were randomized both within and across subjects.

Once a delay-and-duration condition was established, the subject was given one of the 50 -vowel lists. He/she was requested to read the list as fast as possible without making errors. The task is simple enough so that this requirement is not demanding, and

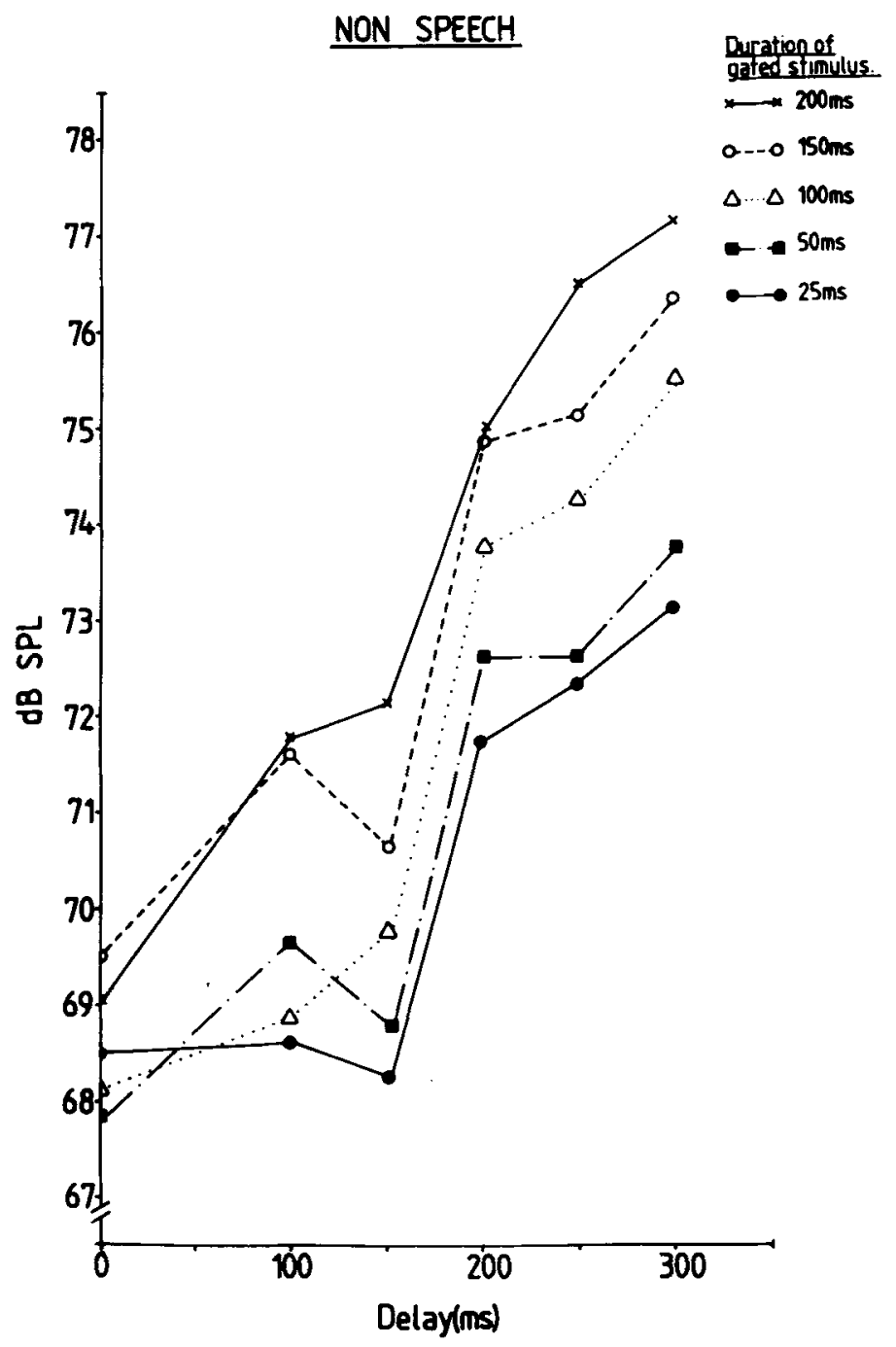

Figure 3. Mean SPL over subjects as a function of delay (in milliseconds) for the subjects who heard nonspeech in the experiment. Delay is along the abscissa and SPL along the ordinate. Connected points come from conditions in which the delayed nonspeech sound had the same duration (the duration can be identified from the symbol labeled in the inset). 
it has the advantage of avoiding the complications of trading accuracy for speed. The subject read the list into the microphone, which, as stated previously, was held at a distance of $20 \mathrm{~cm}$. The experimenter monitored the speech and if an error was made, the subject was given a different list and asked to try again at the same delay and with a sound of the same duration. This was necessary less than $5 \%$ of the time, and on no occasion did a speaker have to read under any delay and duration condition more than twice before performing successfully.

The other measure taken was sound pressure level (SPL, obtained from a Brïel \& Kjaer Sound Pressure Level Type 2203 meter with a B weighting). The microphone of the meter was positioned at a distance of $20 \mathrm{~cm}$ from the speaker and the experimenter read the peak value reached within each list. This was necessary because differences in voice level within and across speakers affect performance time (see the introduction).

\section{RESULTS}

The mean SPLs across delays are shown in Figures 2 and 3 for the speech and nonspeech conditions, respectively. The connected points represent conditions in which the sounds were of the same duration; the delays are given on the abscissas. For each type of material (and for all stimulus durations), SPL increased with increasing delay. In addition, and again this appears to apply to conditions employing both types of material, SPL increased as the duration of the delayed sound increased. These findings differ somewhat from other reported data (usually, as stated in the introduction, an asymptotic function is found). The reason for this discrepancy is probably that the maximum delay employed here was only $300 \mathrm{msec}$.

As noted, differences in voice level across conditions can affect the amount of time needed to read a list (Howell $\&$ Archer, 1984; Howell et al., 1983). The PT data were analyzed by analysis of covariance so that the effects of level (the covariate) were removed. The factors in the analysis were materials (speech or nonspeech, a betweengroups factor), delay $(0,100,150,200,250$, and $300 \mathrm{msec})$, and duration of the delayed segment $(200$, $150,100,50$, and $25 \mathrm{msec}$ ). The mean PTs across delays, after removal of the effects of level for different durations of the delayed portion, are shown in Figures 4 and 5 for speech and nonspeech, respectively.

These data show that the disturbance function of the group of subjects that heard nonspeech after a delay was similar in form to that of the subjects who heard speech. This was supported by the analysis of covariance, as no statistically significant differences between the groups occurred. The functions over delay were peaked, and the peak occurred at a delay of around $200 \mathrm{msec}$ for all durations of the delayed sound. The peaked form of the function was assessed by analysis of the delay effect. The delay effect was significant $[F(5,69)=16.28, p<.0001]$, and the parabolic form of the function was indicated by the highly significant effect of the quadratic term when an orthogonal breakdown was performed $[F(1,13)=$ $55.69, p<.0001]$. These analyses show that subjects responded similarly to delayed speech and nonspeech, and

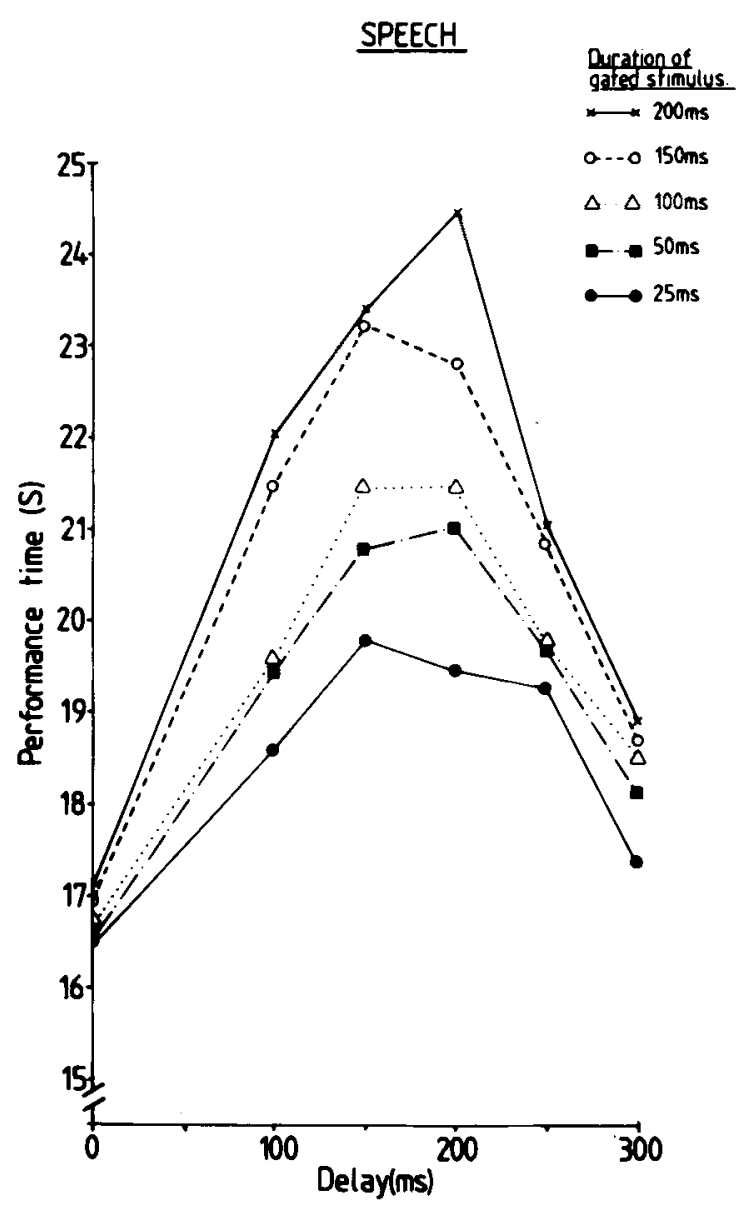

Figure 4. Mean performance time (in seconds) over subjects as a function of delay (in milliseconds) for the subjects who heard speech in the experiment. Delay is along the abscissa and performance time along the ordinate. Connected points come from conditions in which the delayed speech sound had the same duration (the duration can be identified from the symbol labeled in the inset).

that the peaked form of the'disturbance function that is usually reported occurred.

It was predicted that if speakers employed the strategy of waiting for the delayed sound to finish before commencing on the next stretch of speech, disturbance should vary with the duration of the delayed portion. This happened, as inspection of the figures shows. In the analysis of covariance, there was a significant effect of duration of the stimulus $[F(4,55)=57.6, p<.0001]$. Performance time increased systematically with duration of the delayed sound. There appeared to be little (if any) alteration in the location of the peak. This supports the view that there is something especially disruptive about a sound that starts at the point where the speech of the speaker drops in intensity (i.e., sounds that start just after a speaker has completed a syllable are the most disruptive to performance). Although delays of around $200 \mathrm{msec}$ are the most disruptive for all delayed sound durations, there are 


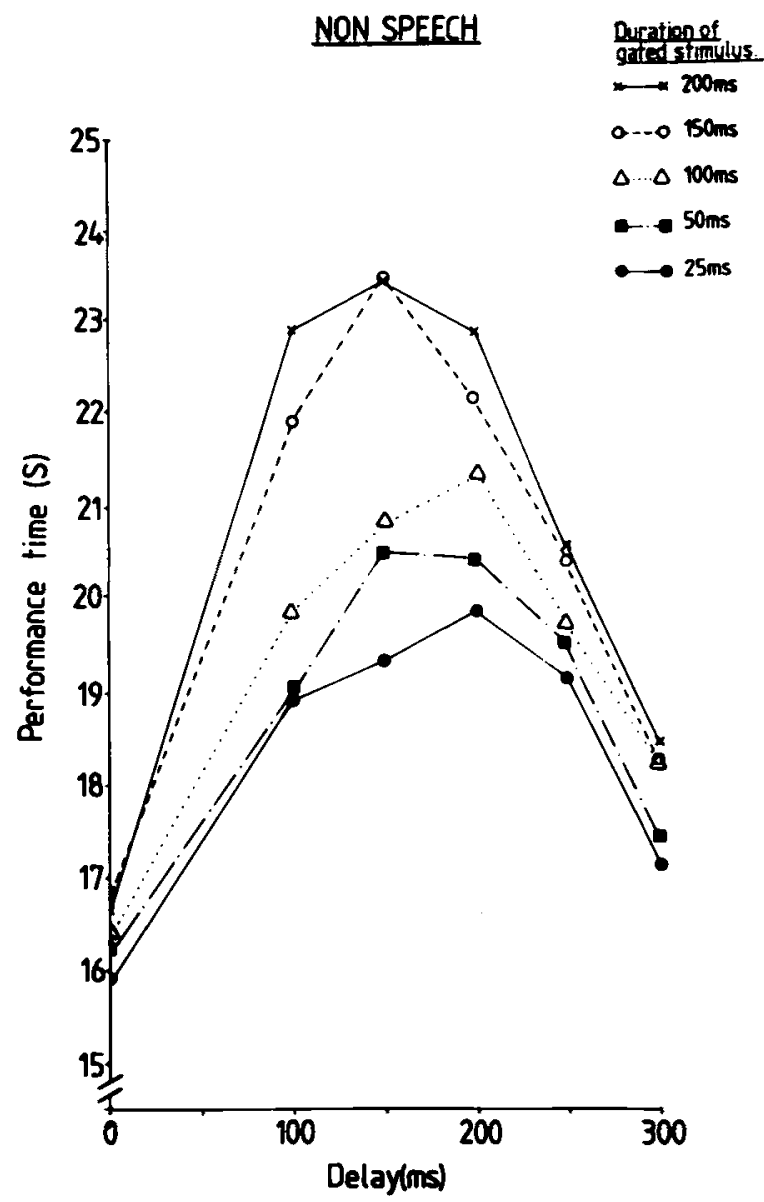

Figure 5. Mean performance time (in seconds) over subjects as a function of delay (in milliseconds) for the subjects who heard nonspeech in the experiment. Delay is along the abscissa and performance time along the ordinate. Connected points come from conditions in which the delayed nonspeech sound had the same duration (the duration can be identified from the symbol labeled in the inset).

differences in the disturbance function for different durations of the delayed sound. In particular, the disturbance functions are less peaked when the delayed section is short. This was shown in the analysis of covariance by a significant interaction between delay and level $[F(20,279)=17.78, p<.0001]$, which, on orthogonal breakdown, showed a strong effect of the quadratic component of delay with the linear component of duration $[F(1,13)=92.75, p<.0001]$.

\section{DISCUSSION}

In brief, our interpretation of the present findings is that the peaked disturbance function on time occurs whether the delayed sound is speech or a nonspeech triangular waveform. When the delayed sound is truncated, the function still peaks at the same delay, although the peak is reduced as duration decreases.
Taking the speech-nonspeech equivalence first, this undermines a feedback-control explanation of the effects of DAF: The nonspeech sound cannot be used for feedback control of speech, yet it produces the same effects as DAF of speech. Thus, the disturbance under DAF must occur for some other reason. Indirectly, the experiment offers support for Howell et al.'s (1983) account of the effects of DAF; thus, the important factor that determines what disruption will occur is where a sound occurs, relative to its intrinsic rhythm, rather than whether the sound is speech or not.

The variation in the amount of disturbance with the duration of the delayed component is consistent with the speaker's adopting a strategy of waiting for the delayed component to finish before proceeding with another sound. On this account, the longer the sound, the longer the speaker would have to wait. One factor introduced into the interpretation of this strategy is that waiting occurs relative to the syllable. This is justified by the fact that syllables specify the intensity variation in speech, and speakers would naturally adopt a strategy relative to some feature in speech that signifies the start and end of variations in intensity of speech. This does not, of course, imply that the syllable has some preeminence as an articulatory control unit, only that intensity varies with this unit.

Taken together, the strategic factors and speechnonspeech equivalence indicate that studies of the effects of DAF have little significance for accounts of speech control that hold that the auditory feedback route is used to check that speech is progressing correctly. In turn, where these explanations have been applied to practical uses of DAF (such as in the treatment of stuttering), reappraisal is required.

\section{REFERENCES}

BACHRACH, D. L. (1964). Sex differences in reactions to delayed auditory feedback. Perceptual \& Motor Skills, 19, 81-82.

BLACK, J. W. (1951). The effect of delayed sidetone upon vocal rate and intensity. Joumal of Speech \& Hearing Disorders, 16, 56-60.

FANT, G. (1959). Acoustic analysis and synthesis of speech with applications to Swedish. Ericsson Technics, 15, 3-108.

Howell, P., \& ARCHER, A. (1984). Susceptibility to the effects of delayed auditory feedback. Perception \& Psychophysics, 36, 296-302.

Howell, P., Powell, D. J., \& KHAN, I. (1983). The amplitude contour of the delayed signal and interference in delayed auditory feedback tasks. Joumal of Experimental Psychology: Human Perception \& Performance, 9, 772-784.

MACKAY, D. G. (1970). Why does language familiarity influence stuttering under delayed auditory feedback? Perceptual \& Motor Skills, 30, 655-669.

Siegel, G. M., Fehst, C. A., Garber, S. R., \& Pick, H. L. (1980). Delayed auditory feedback with children. Journal of Speech \& Hearing Research, 23, 802-813.

(Manuscript received April 10, 1986; revision accepted for publication January 30, 1987.) 